\title{
Modifying the Adsorption Characteristic of Inert Silica Films by Inserting Anchoring Sites
}

\author{
Stefan Ulrich, Niklas Nilius, ${ }^{*}$ and Hans-Joachim Freund \\ Fritz-Haber-Institut der MPG Berlin, Berlin, Germany \\ Umberto Martinez, Livia Giordano, and Gianfranco Pacchioni ${ }^{\dagger}$ \\ Dipartimento di Scienza dei Materiali, Università di Milano-Bicocca, Milano, Italy \\ (Received 14 August 2008; published 8 January 2009)
}

\begin{abstract}
The adsorption properties of thin silica films on Mo(112) have been tailored by embedding single Pd atoms into the nanopores of the oxide material. The embedded $\mathrm{Pd}$ is able to anchor metal adatoms that would not bind to the inert silica surface otherwise. Several adsorption structures, e.g., Pd-Pd, Ag-Pd, and Au-Pd complexes, have been prepared in this way and analyzed with the STM and density functional theory. The binding strength of the different adatoms to the surface is determined by the number of electrons in their frontier orbitals, which introduce a repulsive interaction with the oxide electronic states and weaken the covalent bond to the Pd anchor.
\end{abstract}

DOI: 10.1103/PhysRevLett.102.016102

PACS numbers: 68.43.Fg, 68.37.Ef, 68.47.Gh, 71.15.Mb

Tailoring the adsorption properties of solid materials has been a long-term objective in surface science [1-3]. The functionalization of surfaces is determined by several aspects. First, the density and spatial arrangement of binding sites need to be controlled. Second, the adsorption behavior should be chemically selective, admitting only certain species to interact with the surface. And last, the internal structure of the ad-material should be tunable. Systems with designable adsorption properties have a wide range of applications, e.g., as catalysts in stereoselective reactions, chemical sensors, and template surfaces for selforganization processes.

Several approaches have been developed to fabricate tunable adsorption systems; however, most of them are unable to fulfill all the specifications mentioned above. Regular arrays of binding sites are produced on vicinal surfaces [4], molecular template structures [5], and epitaxial systems exhibiting an ordered network of dislocations $[6,7]$. Chemical sensitivity is achieved by equipping the surface with suitable functional groups to stimulate binding to the desired adsorbates. Alternatively, the sizeselective adsorption behavior of porous materials, e.g., zeolites, is exploited to tune surface reactions via the adsorbate diameter $[1,8]$. Controlling the internal structure of the ad-material remains the most difficult part. Here, mainly atomic manipulation with the scanning tunneling microscope (STM) has been employed to assemble aggregates with a defined structure and chemical composition $[9,10]$.

In this Letter, we report a new approach towards a functionalized adsorption system that is based on the insertion of defined binding sites into an initially inert surface. A suitable system to realize this concept is a porous silica film prepared on $\operatorname{Mo}(112)[11,12]$. The film is chemically inactive and only physisorbs inorganic molecules, like $\mathrm{CO}$ and $\mathrm{H}_{2} \mathrm{O}$ [13]. However, small metal atoms can be inserted into the oxide nanopores [14], as demonstrated for Pd and Ag, whereas larger atoms such as Au are unable to penetrate the holes in the silica top layer $[15,16]$. The inserted atoms remain close to the surface and might be used to stabilize adsorbates on the inert oxide film.

The approach is similar to the doping of oxide materials with impurity atoms or point defects, as an alternative route to creating surface binding sites. Substantial changes in the adsorption behavior were indeed reported for $\mathrm{Li}$ - or $\mathrm{Ti}$ doped $\mathrm{SiO}_{2}$, and $\mathrm{MgO}$ surfaces containing oxygen vacancies $[14,17-19]$. However, while doping affects the global electronic and chemical properties of the oxide material, the insertion of single-atom anchors modifies the binding characteristics only locally. The possibility of using Pd atoms incorporated into a silica film to anchor metal adatoms is now demonstrated with a STM and density functional theory (DFT), approving this new route towards a functionalized adsorption system.

The experiments are performed in a custom built UHVSTM operated at $10 \mathrm{~K}$. The silica film is prepared on an oxygen precovered $\mathrm{Mo}(112)$ surface by depositing 1.2 monolayers silicon at $800 \mathrm{~K}$ in an $\mathrm{O}_{2}$ ambience of $1 \times$ $10^{-7}$ mbar $[11,12]$. Sample annealing to $1200 \mathrm{~K}$ leads to the formation of a crystalline $\mathrm{SiO}_{2}$ layer, as indicated by the sharp $c(2 \times 2)$ LEED pattern [12]. The film is composed of a network of corner-sharing $\mathrm{SiO}_{4}$ tetrahedrons, whereby every $\mathrm{Si}$ atom is connected via bridging $\mathrm{O}$ atoms to three Si neighbors in the plane and to the Mo surface underneath [Fig. 1(a), inset]. The resulting honeycomb structure consists of $-\mathrm{Si}-\mathrm{O}-$ hexagons enclosing a hole of 3-4 $\AA$ diameter that gives access to a nanopore at the $\mathrm{Mo}-\mathrm{SiO}_{2}$ interface. The main structural defects of the film are [110]-oriented domain boundaries, caused by the lateral displacement of two oxide patches by a Mo unit-cell 


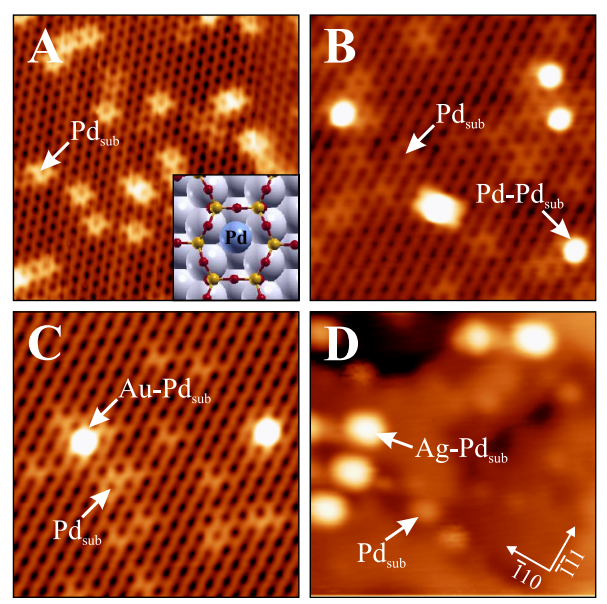

FIG. 1 (color online). (a) STM topographic image of $\mathrm{SiO}_{2} / \mathrm{Mo}(112)$ after insertion of $\mathrm{Pd}_{\text {sub }}$ species $\left(U_{s}=1.2 \mathrm{~V}\right.$, $14 \times 14 \mathrm{~nm}^{2}$ ). (b) Same surface after deposition of single Pd $(0.5 \mathrm{~V})$, (c) $\mathrm{Au}(0.5 \mathrm{~V})$, and (d) Ag atoms (1.9 V, $\left.14 \times 14 \mathrm{~nm}^{2}\right)$. While only $\mathrm{Pd}_{\text {sub }}$ species are observed in (a), new ad-structures become visible in (b-d). The inset in (a) shows a structure model of a $\mathrm{Pd}_{\text {sub }}$ in a silica pore ( $\mathrm{Si}$, small yellow; O, small red; and Mo, large blue circles).

vector (Fig. 2, dashed arrows). Along such boundaries, the regular hexagons are replaced by larger $-\mathrm{Si}-\mathrm{O}-$ octagons alternating with $-\mathrm{Si}-\mathrm{O}-$ squares.

Single $\mathrm{Pd}, \mathrm{Ag}$, and $\mathrm{Au}$ atoms are deposited onto the film from high-purity metal wires $(99.95 \%)$ wrapped with a tungsten filament. Although deposition is performed at $20 \mathrm{~K}$, the atoms have sufficient initial energy to perform transient diffusion into their equilibrium configuration. The local density of states (LDOS) of the sample is probed by differential conductance $(d I / d V)$ spectroscopy performed with lock-in technique and open feedback loop $(10 \mathrm{mV} / 1137 \mathrm{~Hz}$ modulation). DFT calculations are carried out with the generalized gradient approximation using the PW91 exchange-correlation functional and a plane wave basis set (400 eV energy cutoff) as implemented in the VASP code $[20,21]$. The electron-ion interaction is included by the projector-augmented-waves method [22].
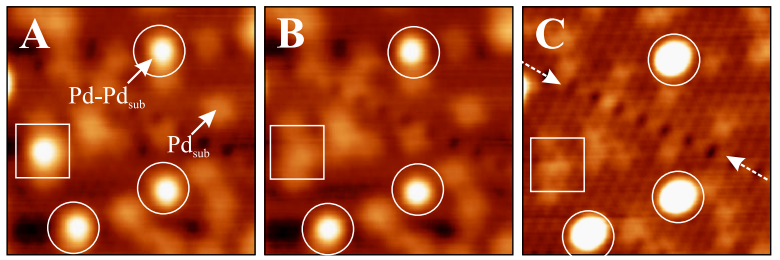

FIG. 2 (color online). (a) STM topographic image of $\mathrm{Pd}_{\text {sub }}$ and $\mathrm{Pd}-\mathrm{Pd}_{\text {sub }}$ species on $\mathrm{SiO}_{2} / \mathrm{Mo}(112)\left(U_{s}=1.0 \mathrm{~V}, 10 \times 10 \mathrm{~nm}^{2}\right)$. (b) As in (a) but after controlled removal of the adatom marked by the square box. (c) As in (b) but with enhanced contrast to show the silica atomic structure. Below the removed atom, the signature of a $\mathrm{Pd}_{\text {sub }}$ becomes visible that has initially anchored the ad-species. The circles indicate other $\mathrm{Pd}-\mathrm{Pd}_{\text {sub }}$ complexes as guides for the eye; the dashed arrows mark a silica domain boundary.
The Mo(112) substrate is described by seven Mo layers, whereby the three bottom layers are kept frozen at bulk positions during geometry optimization. The silica film is modeled with $(4 \times 2)$ and $(6 \times 4)$ supercells, corresponding to a stoichiometry of $\mathrm{Mo}_{14} \mathrm{Si}_{2} \mathrm{O}_{5}$ and $\mathrm{Mo}_{24} \mathrm{Si}_{6} \mathrm{O}_{15}$, respectively.

STM topographic images of the bare film show the characteristic honeycomb structure of the silica. Upon Pd exposure, distinct hexagonal stars appear in images taken at $0.5 \mathrm{~V}$ [Fig. 1(a)]. They transform into bright protrusions of $0.7 \AA$ height above $2.0 \mathrm{~V}$ sample bias. According to earlier studies [16], the features are assigned to Pd atoms that have penetrated the silica nanopores and bind to the Mo- $\mathrm{SiO}_{2}$ interface with $3.3 \mathrm{eV}$ (referred to as $\mathrm{Pd}_{\text {sub }}$ ). The characteristic starlike contrast originates from a hybridization between the $\mathrm{Pd} 5 s$ and $\mathrm{O} 2 p$ orbitals, which renders the oxygen atoms of the hosting $-\mathrm{Si}-\mathrm{O}-$ ring visible in the STM. Above $+2.0 \mathrm{~V}$ sample bias, the Pd $5 s$ becomes directly accessible for tunneling electrons, and the contrast localizes at the $\mathrm{Pd}_{\text {sub }}$ atom in the center of the pore [16].

To investigate the possibility of anchoring single atoms to these $\mathrm{Pd}_{\text {sub }}$ species, three sets of experiments are performed: (i) deposition of $1 \times 10^{13} \mathrm{Pd}$ atoms per $\mathrm{cm}^{2}$ onto a silica film already containing $2 \times 10^{13} \mathrm{~cm}^{-2}$ $\mathrm{Pd}_{\text {sub }}$ and deposition of (ii) $1 \times 10^{12} \mathrm{~cm}^{-2} \mathrm{Au}$ and (iii) $1 \times 10^{12} \mathrm{~cm}^{-2} \mathrm{Ag}$ atoms onto a silica film with $1 \times$ $10^{13} \mathrm{~cm}^{-2} \mathrm{Pd}_{\text {sub }}$ incorporated [Figs. 1(b)-1(d)]. In all three cases, new species appear on the oxide surface, which clearly differ in height, shape, and bias-dependent contrast from the $\mathrm{Pd}_{\text {sub }}$ anchors. The species are imaged as round protrusions at all sample biases, in contrast to the hexagonal shape of inserted $\mathrm{Pd}_{\text {sub }}$, and reach apparent heights of more than $2.0 \AA$ (compared to $0.7 \AA$ for $\mathrm{Pd}_{\text {sub }}$ ). They are not compatible with single $\mathrm{Ag}$ or $\mathrm{Au}$ atoms on the silica surface, either. According to DFT calculations, both atomic species only weakly interact with the oxide film (adsorption energies below $0.05 \mathrm{eV}$ ) and perform rapid diffusion even at low temperature $[14,16]$. Whereas the $\mathrm{Ag}$ atom is able to pass the opening in the $-\mathrm{Si}-\mathrm{O}-$ hexagons, producing similar STM features as the $\mathrm{Pd}_{\text {sub }}$, the $\mathrm{Au}$ atom is too big for penetration and binds exclusively at domain boundaries exposing larger pores. As the new species are neither located along such boundaries nor exhibit the unusual contrast of an embedded atom, they can safely be discriminated from $\mathrm{Ag}$ and $\mathrm{Au}$ atoms. The adsorption structures emerging after $\mathrm{Pd}, \mathrm{Ag}$, and $\mathrm{Au}$ deposition are therefore assigned to single atoms bound to $\mathrm{Pd}_{\text {sub }}$ anchors in the silica film, a hypothesis that will be supported by further experimental evidence in the following.

The ad-species are easily removed from the oxide surface by applying moderate voltage pulses to the STM tip

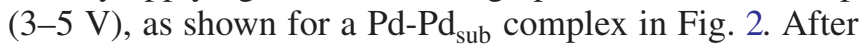
the manipulation experiment, the characteristic signature of an embedded $\mathrm{Pd}_{\text {sub }}$ becomes visible in every case, suggesting that the $\mathrm{Pd}_{\text {sub }}$ anchor is indeed required to stabilize the adatom. Furthermore, the different ad-species exhibit $d I / d V$ fingerprints that clearly deviate from the spectro- 
scopic response of the inserted atoms. For example, while a $\mathrm{Pd}_{\text {sub }}$ shows a weak $d I / d V$ shoulder at $+2.3 \mathrm{~V}$, attributed to the $\mathrm{Pd} 5 s$ orbital [16], a pronounced maximum at $+1.75 \mathrm{~V}$ is observed for the Pd-Pd $\mathrm{d}_{\text {sub }}$ complex [Fig. 3(a)]. Similarly, the $\mathrm{Au}-\mathrm{Pd}_{\text {sub }}$ species exhibits enhanced $d I / d V$ intensity at $-0.2 \mathrm{~V}$ and $+1.6 \mathrm{~V}$ (Fig. 4), whereas no maxima are revealed for $\mathrm{Au}$ monomers located at the domain boundaries. Only for $\mathrm{Ag}-\mathrm{Pd}_{\text {sub }}$ complexes, no $d I / d V$ spectra could be acquired, because the $\mathrm{Ag}$ atom easily desorbs during a spectroscopic run.

The attachment of $\mathrm{Pd}, \mathrm{Ag}$, and $\mathrm{Au}$ atoms to $\mathrm{Pd}_{\text {sub }}$ anchors in the silica film is verified by DFT calculations. In all three examples, the adatoms form covalent bonds with a $\mathrm{Pd}_{\text {sub }}$ sitting in an oxide pore, however, with rather different binding energies. The strongest interaction is found for Pd adatoms, which bind with $1.16 \mathrm{eV}$ to the $\mathrm{Pd}_{\text {sub }}$ (bond length: $2.66 \AA$ ). Without a surface anchor, the binding strength decreases to $0.35 \mathrm{eV}$, and the atoms spontaneously penetrate the hole in the $-\mathrm{Si}-\mathrm{O}-$ hexagons. The adsorption energy of noble-metal atoms is considerably smaller and amounts to $0.35 \mathrm{eV}$ for $\mathrm{Au}-\mathrm{Pd}_{\text {sub }}$ and $0.19 \mathrm{eV}$ for $\mathrm{Ag}-\mathrm{Pd}_{\text {sub }}$ complexes (virtually no binding without $\left.\mathrm{Pd}_{\text {sub }}\right)$. Surprisingly, the atom-atom bond lengths are with $2.54 \AA$ for $\mathrm{Au}-\mathrm{Pd}_{\text {sub }}$ and $2.85 \AA$ for $\mathrm{Ag}-\mathrm{Pd}_{\text {sub }}$ species not much different from the $\mathrm{Pd}-\mathrm{Pd}_{\text {sub }}$ case and are even close to the values of the corresponding gas-phase dimers $\left(\mathrm{Pd}_{2}, 2.54 \AA\right.$; Au-Pd, $\left.2.68 \AA\right)$. In contrast, the binding energy follows an opposite trend for gas-phase $\left(\mathrm{Pd}_{2}, 0.7 \mathrm{eV} ; \mathrm{Au}-\mathrm{Pd}, 1.4 \mathrm{eV}\right)$ [23,24] and surface complexes.

The apparent contradiction between short interatomic distances and low binding energies of the different adstructures indicates the presence of two competing interaction mechanisms on the silica surface. On the one hand, the adatoms form strong covalent bonds to the $\mathrm{Pd}_{\text {sub }}$ anchors. On the other hand, this attractive contribution is counterbalanced by the Pauli repulsion that is mainly exerted by the filled $2 p$ states of the surface oxygen on the adatom. The repulsion is governed by the spatially most expanded adatom orbital, which is the $5 s$ for Pd and $\mathrm{Ag}$ and
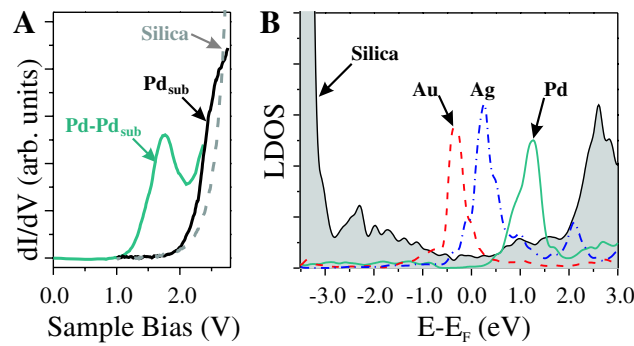

FIG. 3 (color online). (a) $d I / d V$ spectra of a $\mathrm{Pd}_{\text {sub }}$, a $\mathrm{Pd}-\mathrm{Pd}_{\text {sub }}$ complex, and the bare silica surface (set point $2.5 \mathrm{~V}$ ). (b) Calculated state density of the supported silica film (gray region). The line spectra depict the $\mathrm{Pd} 5 s$ (solid line), Ag $5 s$ (dash-dotted line), and Au $6 s$ (dashed line) contribution to the hybrid state formed with the $\mathrm{O} 2 p$ orbitals, when the respective adatom binds to a $\mathrm{Pd}_{\text {sub }}$ anchor. the $6 s$ for the Au. Those frontier orbitals hybridize with the O $2 p$ states upon adsorption, forming new states of antibonding character [Fig. 3(b)]. The strength of the repulsion and therefore the adatom-surface interaction is now controlled by the number of electrons in these hybrid states. The Pd $5 s-\mathrm{O} 2 p$ mixed state is located at $+1.25 \mathrm{eV}$ above the Fermi level $\left(E_{F}\right)$ and therefore empty, which reduces the repulsion with the oxygen states and allows the formation of a strong $\mathrm{Pd}-\mathrm{Pd}_{\text {sub }}$ bond. The binding energy is even higher than in gas-phase $\mathrm{Pd}_{2}$, reflecting the stabilization effect of the Mo support. The position of the $\mathrm{Pd}$ $5 s$-derived state above $E_{F}$ manifests the charge neutrality of the adatom, as the state is empty in gas-phase Pd, too. The hybrid state shows up also experimentally and is assigned to the $d I / d V$ peak at $+1.75 \mathrm{~V}$ observed for

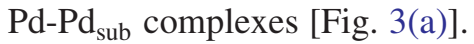

In contrast, the hybrid state formed between the $\mathrm{Ag} 5 s$ (Au 6s) orbital and the O $2 p$ states is singly (doubly) occupied, as deduced from its calculated energy position at $+0.1 \mathrm{eV}(-0.3 \mathrm{eV})$ [Fig. 3(b)]. Because of the vicinity to $E_{F}$, they could not be identified in the $d I / d V$ spectra, although the enhanced signal in conductance maps of $\mathrm{Au}-\mathrm{Pd}_{\text {sub }}$ complexes at $-0.2 \mathrm{~V}$ might indicate tunneling into the $\mathrm{Au} 6 s-\mathrm{O} 2 p$ state (Fig. 4). The electron(s) in the mixed metal-oxygen states induce substantial Pauli repulsion with the silica LDOS, which partly compensates the attractive interaction with the $\mathrm{Pd}_{\text {sub }}$ and gives rise to the small binding energies of $\mathrm{Ag}$ and $\mathrm{Au}$ atoms. The effect is larger for $\mathrm{Au}$, because its valence $s$ orbital is doubly occupied ( $6 s^{2}$ configuration) as a result of a charge transfer from the Mo support. The formation of anionic Au species has been observed for other oxide films before and manifests the strong electronegative character of gold $[25,26]$. Instead, the Ag adatom remains neutral on the silica sur-

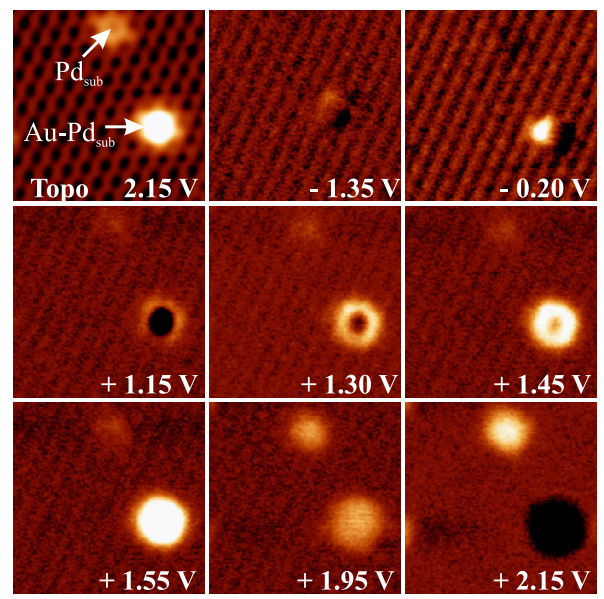

FIG. 4 (color online). Topographic image and $d I / d V$ maps of a $\mathrm{Pd}_{\text {sub }}$ and a $\mathrm{Au}-\mathrm{Pd}_{\text {sub }}$ species on $\mathrm{SiO}_{2} / \mathrm{Mo}(112)$ taken at different sample biases $\left(6 \times 6 \mathrm{~nm}^{2}\right)$. The increase of the $d I / d V$ signal at the $\mathrm{Au}-\mathrm{Pd}_{\text {sub }}$ at -0.2 and $+1.55 \mathrm{~V}$ marks two intrinsic states of the complex, whereas the ringlike enhancement at $1.0-1.5 \mathrm{~V}$ is related to a deformation of the silica lattice upon Au adsorption. 
face, since its $5 s$ orbital carries a single electron as in gasphase Ag. The comparable adsorption energies for Au and $\mathrm{Ag}$ atoms, in spite of different repulsive terms, reflect the balancing influence of the metal $d$ states. Whereas the Au $5 d$ states considerably strengthen the $\mathrm{Au}-\mathrm{Pd}_{\text {sub }}$ interaction, the $\mathrm{Ag} 4 d$ is too low in energy to participate in the bond formation.

To reduce steric repulsion, the $-\mathrm{Si}-\mathrm{O}-$ hexagon hosting the $\mathrm{Pd}_{\text {sub }}$ strongly relaxes upon adatom attachment. The effect is most pronounced for the Au- $\mathrm{Pd}_{\text {sub }}$ complex due to the double occupancy of the Au $6 s-\mathrm{O} 2 p$ hybrid state. According to the DFT calculations, the opening in the $-\mathrm{Si}-\mathrm{O}-$ hexagon enlarges by several tenths of an angstrom $(\sim 5 \%)$ and enables the $\mathrm{Au}$ to approach the $\mathrm{Pd}_{\text {sub }}$ anchor. As a result, the Au binding energy increases from $0.14 \mathrm{eV}$ as calculated for a rigid geometry to $0.35 \mathrm{eV}$ after structural relaxation. The ring widening is accompanied by a reduction of the silica LDOS next to the $\mathrm{Au}-\mathrm{Pd}_{\text {sub }}$ binding site, which reflects the decreasing $\mathrm{Si}-\mathrm{O}$ orbital overlap in the enlarged hexagon. The reduced state density is indeed observed in $d I / d V$ maps of the Au-Pd $d_{\text {sub }}$ complex taken between 1.0 and $1.5 \mathrm{~V}$ sample bias (Fig. 4). To balance the outward relaxation of the hexagon, $\mathrm{Si}-\mathrm{O}$ bonds need to be compressed in a circular region surrounding the adsorption site. The resulting increase of the oxide LDOS is also visible in $d I / d V$ maps, and shows up as a ringlike intensity enhancement around the $\mathrm{Au}-\mathrm{Pd}_{\text {sub }}$ species. The ring diameter is approximated with $10 \mathrm{~A}$, indicating that mainly the next shell of $-\mathrm{Si}-\mathrm{O}-$ hexagons is affected by the compressive stress exerted by the enlarged silica pore. Similar ring structures are also observed for the $\mathrm{Pd}-\mathrm{Pd}_{\mathrm{sub}}$ complexes.

In conclusion, $\mathrm{Pd}_{\text {sub }}$ atoms incorporated into the nanopores of a thin silica film are able to anchor various adatoms at the originally inert oxide surface. The stabilization effect results from a strong covalent bonding between adatom and embedded $\mathrm{Pd}_{\text {sub }}$, which is, however, partly compensated by the Pauli repulsion of the filled $2 p$ states of the surface oxygen atoms. The strength of the repulsive interaction is dominated by the occupancy of the frontier orbitals of the different adatoms and increases when going from Pd with an empty valence $s$ state, to neutral Ag and negatively charged Au species with singly and doubly occupied $s$ orbitals, respectively. The ratio between repulsive and attractive terms in the anchoring process thus depends on the electronic properties of the adatoms, introducing a certain chemical sensitivity of the interaction mechanism. The approach described here can be employed to produce even more complex structures via subsequent attachment of different atomic species to the surface anchors. The successful insertion of single-atom binding sites into porous silica films on $\mathrm{Mo}(112)$ therefore presents a first step towards the preparation of a functionalized adsorption system.
This Letter has been supported by COST Action D41. Part of the computing time was provided by the Barcelona Supercomputing Center (BSC-CNS).

*Corresponding author. nilius@fhi-berlin.mpg.de

Corresponding author. gianfranco.pacchioni@unimib.it

[1] H. Li, M. Eddaoudi, M. O`Keeffe, and O. M. Yaghi, Nature (London) 402, 276 (1999).

[2] Functionalization of Nanomaterials, edited by C.S.R. Kumar (Wiley-VCH, Weinheim, 2005).

[3] H. J. Freund, Surf. Sci. 601, 1438 (2007).

[4] N. Neel, J. Kröger, and R. Berndt, Appl. Phys. Lett. 88, 163101 (2006).

[5] J.A. Theobald, N.S. Oxtoby, M.A. Phillips, N.R. Champness, and P. H. Beton, Nature (London) 424, 1029 (2003).

[6] H. Brune, M. Giovannini, K. Bromann, and K. Kern, Nature (London) 394, 451 (1998).

[7] C. Becker et al., New J. Phys. 4, 75 (2002).

[8] S. Bhatia, Zeolite Catalysis: Principles and Applications (CRC Press, Boca Raton, FL, 1990).

[9] N. Nilius, T.M. Wallis, and W. Ho, Science 297, 1853 (2002).

[10] G. V. Nazin, X.H. Qui, and W. Ho, Science 302, 77 (2003).

[11] T. Schroeder et al., Surf. Rev. Lett. 7, 7 (2000).

[12] J. Weissenrieder et al., Phys. Rev. Lett. 95, 076103 (2005).

[13] J. B. Giorgi, T. Schröder, M. Bäumer, and H.-J. Freund, Surf. Sci. Lett. 498, L71 (2002).

[14] L. Giordano, A. Del Vitto, and G. Pacchioni, J. Chem. Phys. 124, 034701 (2006).

[15] M. Baron et al., J. Phys. Chem. C 112, 3405 (2008).

[16] S. Ulrich, N. Nilius, H.-J. Freund, U. Martinez, L. Giordano, and G. Pacchioni, Chem. Phys. Chem. 9, 1367 (2008).

[17] J. X. Wang and J. H. Lunsford, J. Phys. Chem. 90, 5883 (1986).

[18] M. C. Wu, C. M. Truong, and D. W. Goodman, Phys. Rev. B 46, 12688 (1992); B. K. Min, W. T. Wallace, and D. W. Goodman, J. Phys. Chem. B 108, 14609 (2004).

[19] M. Sterrer et al., Angew. Chem., Int. Ed. 45, 2630 (2006).

[20] J. P. Perdew et al., Phys. Rev. B 46, 6671 (1992).

[21] G. Kresse and J. Hafner, Phys. Rev. B 47, 558 (1993); G. Kresse and J. Furthmüller, Phys. Rev. B 54, 11169 (1996).

[22] P. E. Blöchl, Phys. Rev. B 50, 17953 (1994).

[23] D. Andrea, U. Haussermann, M. Dolg, H. Stoll, and H. Preuss, Theor. Chim. Acta 78, 247 (1991); P. Schwerdtfeger, Chem. Phys. Lett. 183, 457 (1991); J. Ho, K. M. Ervin, M. L. Polak, M. K. Gilles, and W. C. Lineberger, J. Chem. Phys. 95, 4845 (1991).

[24] Handbook of Chemistry and Physics, edited by D. R. Lide (CRC Press, Boca Raton, FL, 1998).

[25] M. Sterrer et al., Phys. Rev. Lett. 98, 096107 (2007).

[26] N. Nilius et al., Phys. Rev. Lett. 100, 096802 (2008). 